\title{
SUPPORT THEOREMS FOR RADON TRANSFORMS ON REAL ANALYTIC LINE COMPLEXES IN THREE-SPACE
}

\author{
JAN BOMAN AND ERIC TODD QUINTO
}

\begin{abstract}
In this article we prove support theorems for Radon transforms with arbitrary nonzero real analytic measures on line complexes (three-dimensional sets of lines) in $\mathbb{R}^{3}$. Let $f$ be a distribution of compact support on $\mathbb{R}^{3}$. Assume $Y$ is a real analytic admissible line complex and $Y_{0}$ is an open connected subset of $Y$ with one line in $Y_{0}$ disjoint from supp $f$. Under weak geometric assumptions, if the Radon transform of $f$ is zero for all lines in $Y_{0}$, then $\operatorname{supp} f$ intersects no line in $Y_{0}$. These theorems are more general than previous results, even for the classical transform. We also prove a support theorem for the Radon transform on a nonadmissible line complex. Our proofs use analytic microlocal analysis and information about the analytic wave front set of a distribution at the boundary of its support.
\end{abstract}

\section{INTRODUCTION}

A generalized Radon transform on lines in space, $R_{\mu}$, integrates functions on $\mathbb{R}^{3}$ over lines using a specified integration weight, $\mu$, on each line. For compactly supported functions, injectivity for this transform with positive $C^{2}$ weights [5] follows from local invertibility for Radon transforms on lines in a plane (e.g., [16]). Injectivity follows from the plane result because line integrals over all lines in space provide line integrals over lines in any given plane. Injectivity of this transform can be reduced to a plane problem because this transform is overdetermined: the set of lines in space has greater dimension than $\mathbb{R}^{3}$. As the transform is overdetermined, it is intriguing to find threedimensional submanifolds, $Y$, of lines, the so-called line complexes, for which $R_{\mu}$ restricted to $Y$ is invertible.

The so-called admissible complexes are: the set of lines tangent to a surface in space, the set of lines intersecting a curve in space, and the set of lines with direction vectors lying on a given curve on the sphere $[6,7]$. All of these complexes have similar geometric properties as reflected in Gelfand's admissibility condition (3.1.3).

Received by the editors July 16, 1990 and, in revised form, November 19, 1990.

1991 Mathematics Subject Classification. Primary 44A12; Secondary 58G15.

Key words and phrases. Radon transform, admissible line complex, analytic microlocal analysis.

The first author is grateful to the Office of the Provost of Tufts University and to the Royal Swedish Academy of Sciences for their support. He was also partially supported by Swedish Natural Sciences Research Council grant F-FU4047-110. The second author thanks the Swedish Natural Sciences Research Council for its support. He was also partially supported by NSF grants MCS 8701415 and MCS 8901203 . Both authors are grateful to the referee for helpful comments and a reference. 
In this article we prove injectivity and, more importantly, support theorems for real analytic admissible line complexes of real lines in $\mathbb{R}^{3}$ with arbitrary real analytic weights that are never zero. These support theorems guarantee uniqueness for tomography with sources on a curve. Counterexamples are given that show the theorems do not hold under weaker hypotheses. Our proofs are in the spirit of Boman and Quinto [2, 3] and involve the theory of analytic Fourier integral operators, microlocal analysis, and a theorem [13] about the analytic wave front set of a distribution at points on the boundary of its support. Even for the classical transform and real analytic manifolds, our theorems are stronger than previous results. Our theorems can be generalized to many other Radon transforms (e.g., [18]), and the cases considered here exhibit the important ideas and are of some practical interest.

In Example 4.3 we prove injectivity on functions of compact support for a Radon transform on a nonadmissible complex $Y$ with arbitrary nowhere zero real analytic measures. We also prove Helgason's support theorem for this transform. Guillemin [10] discusses microlocal advantages of admissibility, so it is not surprising that the microlocal analysis is more difficult for nonadmissible complexes and that the theorem is weaker. This example (with canonical measures) was described (without proof) in the complex category by Gelfand and Graev [6] (see also Greenleaf and Uhlmann [9, pp. 231-237] for related results for $\mathbb{R}^{3}$ ).

Gelfand and Graev [6] have proven injectivity for admissible complexes of complex lines in $\mathbb{C}^{3}$ with standard weights and where the curves and surfaces are algebraic. Guillemin and Sternberg [11] first considered the Radon transform as a Fourier integral operator (see also [17]), and Guillemin [10] investigated admissiblity using microlocal analysis. Using the calculus of Fourier integral operators, Greenleaf and Uhlmann [9] considered admissible complexes of real geodesics on symmetric spaces in the $C^{\infty}$ category and proved that $R_{\mu}^{*} R_{\mu}$ is in a class of Fourier integral operators associated with intersecting Lagrangian manifolds. For the transform with $\mu$ a nonzero constant and for the complex of lines through a curve, Solmon [20] proved a support theorem and Finch [4] developed an inversion method. See [12] for a proof of injectivity for the Radon transform with constant weights on all geodesics on symmetric spaces.

Section 2 of this article provides the important terms and the main theorems. Proofs are done in $\S 3$, and the counterexamples are given in $\S 4$.

\section{DEFINITIONS AND MAIN THEOREMS}

For $\theta \in \mathbf{S}^{2}$ and $x \in \mathbb{R}^{3}$ let $\ell(\theta, x)=\{x+s \theta \mid s \in \mathbb{R}\}$ be the line containing $x$ and parallel to $\theta$, and let $d s$ be arc length measure on the line. Let $Y$ be an immersed three-dimensional real analytic submanifold of the set of lines in three-space. Such a $Y$ will be called a line complex. Let $X \subset \mathbb{R}^{3}$ be open and let $\mu(x, \ell)$ be a $C^{\infty}$ function on

$$
Z=\{(x, \ell) \in X \times Y \mid x \in \ell\} .
$$

The generalized Radon transform with weight $\mu, R_{\mu}: C_{c}\left(\mathbb{R}^{3}\right) \rightarrow C(Y)$, is defined for $\ell=\ell(\theta, x) \in Y$ by

$$
R_{\mu} f(\ell)=R_{\mu} f(\theta, x):=\int_{z \in \ell(\theta, x)} f(z) \mu(z, \ell(\theta, x)) d s .
$$


If $W$ is a surface in $\mathbb{R}^{3}$ and $\ell$ is a line tangent to $W$ at point of tangency $w_{0}$, we define the directional curvature of $W$ along $\ell$ at $w_{0}$ to be the curvature at $w_{0}$ of the curve of intersection of $W$ with the plane through $\ell$ and parallel to the normal to $W$ at $w_{0}$.

The classical admissible complexes will be labeled as follows:

Type I: Given a nonplanar real analytic surface $W$ in space, $Y$ is the set of all lines, $\ell$, tangent to $W$, such that $W$ has nonzero directional curvature along $\ell$ at every point of tangency.

Type II: Given a real analytic curve $\gamma: \mathbb{R} \rightarrow \mathbb{R}^{3}$ that is nonsingular $\left(\gamma^{\prime}(t) \neq 0 \forall t\right), \quad Y$ is the set of lines intersecting that curve nontangentially.

Type III: $\quad Y$ is the set of lines parallel to directions on a given real analytic curve on the sphere, $\mathbf{S}^{2}$.

The geometric conditions given for type I and II complexes ensure that the sets $Y$ are immersed submanifolds of the set of all lines in $\mathbb{R}^{3}$. Our theorems are easier to state for subsets $Y_{0} \subset Y$, which are imbedded in the set of all lines. For subsets of type I complexes, this implies that each line in $Y_{0}$ is tangent to $W$ in exactly one point. For subsets of type II complexes, this implies that the curve is simple and that no line in $Y_{0}$ intersects the curve at more than one point. As our theorems are local, they can be applied locally to the $Y_{0}$ that are not imbedded. We shall not consider the complex of lines in a one-parameter family of planes; this complex is similar to the complex of lines tangent to a surface.

The main theorems are now given.

Theorem 2.1. Let $W$ be a nonplanar real analytic surface in $\mathbb{R}^{3}$. Let $Y_{0}$ be an open connected subset of the type I complex defined by $W$, and assume $Y_{0}$ is an imbedded submanifold of the set of all lines. In case there is a plane $\mathscr{P}$ tangent to $W$ at a nondiscrete set of points, we assume no line in $Y_{0}$ is contained in $\mathscr{P}$. Let $X$ be an open set in $\mathbb{R}^{3}$ disjoint from $W$, and let $\mu(x, \ell)$ be a real analytic function on $Z$ (see (2.1)) that is never zero. Let $f \in \mathscr{E}^{\prime}(X)$. If $\left.R_{\mu} f\right|_{Y_{0}}=0$ and if some line in $Y_{0}$ is disjoint from supp $f$, then every line in $Y_{0}$ is disjoint from supp $f$.

If $W$ is strictly positively curved, then the geometric assumptions in the theorem hold for all lines tangent to $W$ and all functions $f$ with support disjoint from $W$. Example 4.1 shows the necessity of the assumption that no line in $Y_{0}$ (that intersects supp $f$ ) is contained in a plane that is tangent to $W$ at a nondiscrete set of points. This assumption about tangent planes to $W$ is needed only for Proposition 3.2.3. Proposition 3.2.1 is a weaker support theorem that is valid without this assumption.

Theorem 2.2. Let $\gamma$ be a nonsingular real analytic curve. Let $Y_{0}$ be an open connected subset of the type II complex defined by $\gamma$, and assume $Y_{0}$ is an imbedded submanifold of the set of all lines. Assume that, if $\gamma$ is a plane curve, then no line in $Y_{0}$ lies in a plane containing $\gamma$. Let $X$ be an open set in $\mathbb{R}^{3}$ disjoint from $\gamma$, and let $\mu(x, \ell)$ be a real analytic function on $Z$ (see (2.1)) 
that is never zero. Let $f \in \mathscr{E}^{\prime}(X)$. If $\left.R_{\mu} f\right|_{Y_{0}}=0$ and if some line in $Y_{0}$ is disjoint from supp $f$, then every line in $Y_{0}$ is disjoint from supp $f$.

Theorem 2.2 is true even if lines tangent to $\gamma$ are included in $Y_{0}$. Example 4.2 shows that the conclusion of Theorem 2.2 is not true if $\gamma$ is a plane curve and $Y_{0}$ contains lines (that intersect $\operatorname{supp} f$ ) in a plane that contains $\gamma$. A type II complex defined by the curve $\gamma$ can be viewed as a limiting case as $\varepsilon \rightarrow 0^{+}$ of type I complexes defined by surfaces of tubes of radius $\varepsilon$ about $\gamma$.

In a very nice article, Solmon [20] proves a result closely related to Theorem 2.2 that is valid for all continuous curves $\gamma$. He assumes that $\mu$ is constant, that $Y_{0}$ is the set of all lines through $\gamma$ with directions parallel a fixed open set $S$ in $\mathbf{S}^{2}$, and that for every direction in $S$ there is a line in $Y_{0}$ in that direction that is disjoint from supp $f$. The conclusion is that every line in $Y_{0}$ is disjoint from $\operatorname{supp} f$. Theorem 2.2 is stronger than Solmon's theorem even for constant $\mu$ because the open set, $S$, of directions is not fixed but can vary with the point $x \in \gamma$. However, Theorem 2.2 does not apply when $\gamma$ is a line and Solmon's theorem does. On the other hand, Proposition 3.3.1 for characteristic paths (3.3.1) with pivot points at infinity (which is true for all analytic curves) implies Solmon's theorem for all analytic curves, including lines.

Theorem 2.3. Let $\theta: \mathbf{S}^{1} \rightarrow \mathbf{S}^{2}$ be a closed simple nonsingular real analytic curve of directions and let $Y_{0}$ be an open connected subset of the type III complex of lines with directions on $\theta\left(\mathbf{S}^{1}\right)$. Assume $\theta\left(\mathbf{S}^{1}\right)$ is not a great circle of $\mathbf{S}^{2}$. Let $\mu(x, \ell)$ be a real analytic function on $Z$ (see (2.1)) that is never zero. Let $f \in \mathscr{E}^{\prime}\left(\mathbb{R}^{3}\right)$. If $\left.R_{\mu} f\right|_{Y_{0}}=0$ and if some line in $Y_{0}$ is disjoint from supp $f$, then every line in $Y_{0}$ is disjoint from supp $f$.

Theorem 2.3 follows from Theorem 2.2 together with the observation that a type III complex can be viewed as a type II complex with the curve at infinity. A similar construction to that in Example 4.2 shows that the conclusion of Theorem 2.3 is not valid if $\theta\left(\mathbf{S}^{1}\right)$ is a great circle (and so $Y$ is the set of lines in parallel slices).

In all of these theorems, $\mu$ only needs to be defined for $x$ in some open set containing $\operatorname{supp} f$.

The proofs use the calculus of the Fourier integral operator $R_{\mu}$ and a theorem of Hörmander [14, Theorem 8.5.6] (quoted in §3.1) on analytic singularities. Let $\ell_{1} \in Y_{0}$ be parallel to $\theta_{1} \in \mathbf{S}^{2}$. The first key idea in the proof involves the microlocal analysis of $R_{\mu}$ : data $R_{\mu} f(\ell)$ for lines $\ell \in Y_{0}$ near $\ell_{1}$ detect microlocal analytic singularities (wave front) of $f$ at points on $\ell_{1}$ in almost all cotangent directions perpendicular to $\theta_{1}$ (Proposition 3.1.1, [9] in the $C^{\infty}$ category). Therefore, if $R_{\mu} f(\ell)=0$ for lines in $Y_{0}$ near $\ell_{1}$ then $f$ must be analytic at all points $x \in \ell_{1} \cap X$ in almost all directions perpendicular to $\theta_{1}$. (This is a slight abuse of notation: precisely, $f$ is analytic at $x$ in direction $\xi$ if $(x, \xi) \notin W F_{A}(f)$, the analytic wave front set of $f$ [14].) The second key idea involves a theorem (given in §3.1) about analytic wave front sets; under certain assumptions, this theorem implies vanishing of a function at $y$ if the function is analytic in a specified direction at $y$. The proofs of our support theorems proceed as follows. Assume supp $f$ does not intersect the line $\ell_{0} \in Y_{0}$ and let $\ell_{1} \in Y_{0}$. Using the assumptions that $Y_{0}$ is open and connected we construct a sequence of characteristic paths of lines in $Y_{0}$ from $\ell_{0}$ to $\ell_{1}$ (for type $I$ 
complexes: Propositions 3.2.1 and 3.2.3). The first key idea about $R_{\mu}$ implies $f$ is analytic in most directions at all points on all lines in the path because $R_{\mu} f$ is zero near these lines. The second key step in the proof is to use [14, Theorem 8.5.6] to show that $f$ must be zero near those lines. This proves the theorem. The proofs are more subtle than those in [3] for the Radon transform on hyperplanes in $\mathbb{R}^{n}$ since, in the case of lines, some important wave front directions of $f$ are not detected by the data $R_{\mu} f$. The heart of the article is $\S \S 3.2-3.3$ in which these more subtle arguments are given.

Using ideas related to those in [3], Globevnik [8] has proved a clever local support theorem for the X-ray transform with $\mu=1$ and data on all lines; similar theorems are true for real analytic transforms on admissible line complexes, and the proofs are local versions of the proofs below.

\section{Proofs}

Throughout this section we assume that $\mu$ is real analytic and never zero and that all manifolds are real analytic. One key to the proofs of all our support theorems is the following important theorem of Hörmander (generalized by Kashiwara and Kawai [15] for hyperfunctions [19]).

Theorem 8.5.6 [14]. Let $X$ be an open subset of $\mathbb{R}^{n}, f \in \mathscr{D}^{\prime}(X)$, and $x_{0}$ a boundary point of the support of $f$, and assume that there is a $C^{2}$ function $F$ such that $F\left(x_{0}\right)=0, d F\left(x_{0}\right) \neq 0$, and $F(x) \leq 0$ on supp $f$. Then $\left(x_{0}, \pm d F\left(x_{0}\right)\right) \in W F_{A}(f)$, where $W F_{A}(f)$ is the analytic wave front set of $f$.

Let $x_{0}$ be a point on the boundary of the surface defined by the function $F$ in Theorem 8.5.6 above, and let $\xi_{0}$ be conormal to that boundary at $x_{0}$. The theorem implies that if $\left(x_{0}, \xi_{0}\right) \notin W F_{A}(f)$, then $x_{0} \notin \operatorname{supp} f$.

3.1. The microlocal analysis of $R_{\mu}$ for admissible complexes. The first key idea of the proofs requires the microlocal analysis of $R_{\mu}$ and it reflects the point of view of Guillemin and Sternberg $[10,11]$ that an understanding of the geometric properties of the double fibration (below left) and the corresponding map on the cotangent spaces (below right) is the appropriate way to discover the microlocal properties of $R_{\mu}$ as a Fourier integral operator:
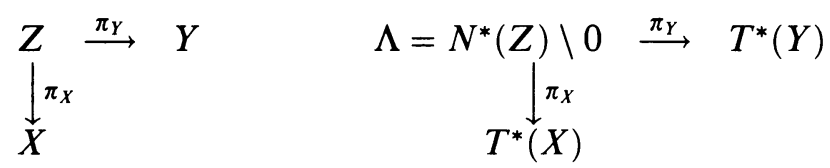

In (3.1.1), $N^{*}(Z)$ is the conormal bundle of $Z$ in $T^{*}(X \times Y)$. The spaces (manifolds or cotangent spaces) to which the projections $\pi_{X}$ and $\pi_{Y}$ apply will be clear from the context. In order that (3.1.1) defines a double fibration, we require the surjectivity condition:

$$
\pi_{X}: Z \rightarrow X \text { has surjective differential. }
$$

If $Y$ is a complex of type I or II then a calculation in local coordinates shows that (3.1.2) implies that $X$ is disjoint from the surface or curve defining $Y$. For $x \in X$ define $\Sigma_{x}=\bigcup \pi_{Y}\left(\pi_{X}^{-1}(x)\right)$, the set of all points on the lines in the complex $Y$ that pass through $x$. Admissibility is defined by Gelfand and 
Graev [6, p. 810] in terms of the $\Sigma_{x}$; admissible complexes of type I, II, and III all satisfy his admissibility condition:

$$
\begin{aligned}
& \text { if } x_{1} \in \ell \in Y \text { and } x_{2} \in \ell, \\
& \text { then } \Sigma_{x_{1}} \text { and } \Sigma_{x_{2}} \text { have the same tangent plane along } \ell .
\end{aligned}
$$

Proposition 3.1.1. Let $Y$ be a real analytic line complex such that the surjectivity (3.1.2) and admissibility (3.1.3) conditions hold for all $x \in X$ and all $\ell \in Y$. Let $\ell_{0} \in Y$ and assume $f \in \mathscr{E}^{\prime}(X)$ and $R_{\mu} f(\ell)=0$ for all $\ell \in Y$ in $a$ neighborhood of $\ell_{0}$. Let $x \in \ell_{0} \cap X$ and let $\xi \in T_{x}^{*}(X)$ be conormal to $\ell_{0}$ but not conormal to the tangent plane to $\Sigma_{x}$ along $\ell_{0}$. Then $(x, \xi) \notin W F_{A}(f)$.

Let $\ell_{0} \in Y_{0}$. For $x \in \ell_{0}$, let $N_{x}^{*}\left(\ell_{0}\right) \subset T_{x}^{*}(X)$ be the conormal space of $\ell_{0}$ at $x$. The conclusion of Proposition 3.1.1 is that the cotangent directions in $N_{x}^{*}\left(\ell_{0}\right)$ that are not detected by $R_{\mu}$ are exactly those conormal at $x$ to the tangent plane to $\Sigma_{x}$ along $\ell_{0}$. By Gelfand's admissibility condition, the undetected cotangent directions are the same for every point on $\ell_{0}$. For type I complexes, these undetected directions are exactly those $\xi$ that are conormal to $W$ at the point of tangency of $\ell_{0}$ with $W$; for type II complexes, they are the $\xi$ that are conormal to the curve $\gamma$ at the point of intersection of $\ell_{0}$ with $\gamma$.

Proposition 3.1.1 is proven for the $C^{\infty}$ category in [9], and the arguments are essentially the same as in the analytic category, so they will only be sketched here. Let $\Lambda_{0}$ be the open subset of $\Lambda$ consisting of all $(x, \xi, \ell, \eta) \in \Lambda$ for which $\xi$ is not conormal to $\Sigma_{x}$ along $\ell_{0}$. Using essentially only the surjectivity and admissibility conditions, one proves: $R_{\mu}$ is a Fourier integral operator with Lagrangian manifold $\Lambda ; \Lambda_{0}$ is a local canonical graph; and $R_{\mu}$ is analytic elliptic when microlocally restricted to $\Lambda_{0}$. One finishes the proof of the proposition by using the calculus of Fourier integral operators to show that $R_{\mu} f=0$ near $\ell$ implies that the specified directions are not in $W F_{A} f$. Finally, one needs to check using local coordinates that complexes satisfying the hypotheses of Theorems 2.1 and 2.2 satisfy the admissibility and surjectivity conditions.

3.2. Characteristic paths for type I complexes and the proof of Theorem 2.1. Let $\ell_{0} \in Y_{0}$ be a line disjoint from $\operatorname{supp} f$, and let $\ell_{1} \in Y_{0}$. We will construct a path in $Y_{0}$ from $\ell_{0}$ to $\ell_{1}$ and show $f$ is zero near all lines in the path. Since the analytic wave front set of $f$ in the exceptional directions $\xi$ conormal to $\Sigma_{x}$ is not detected by data $R_{\mu} f$, "characteristic" paths for $R_{\mu}$ must be constructed for which these directions are not problematic.

Let $x_{0} \in \mathbb{R}^{3} \backslash W$. A characteristic path with pivot point $x_{0}$ is a smooth path of lines in the set $\pi_{Y}\left(\pi_{X}^{-1}\left(x_{0}\right)\right)$, where these maps are defined by the double fibration on the left of (3.1.1). For type I complexes, characteristic paths consist of lines that intersect $x_{0}$ and are tangent to $W$. A characteristic path with pivot point at infinity is a smooth path of parallel lines tangent to $W$.

Proposition 3.2.1. Assume that the hypotheses of Theorem 2.1 hold for $X, W$, and $Y_{0}$. Let $f \in \mathscr{E}^{\prime}(X)$ and assume $R_{\mu} f=0$ on $Y_{0}$. Let $l:[a, b] \rightarrow Y_{0}$ be $a$ characteristic path and assume $l(a)$ does not meet supp $f$ and the pivot point of the path $l(s)$ is disjoint from supp $f$. Then

$$
l(s) \cap \operatorname{supp} f=\varnothing \quad \text { for } a \leq s \leq b .
$$


Proof. We can assume the characteristic path has pivot point at infinity. This reduction uses an affine transformation of $R P^{3} \supset \mathbb{R}^{3}$.

The reduction is as follows. Consider the projective space $R P^{3}$ to be the union of $\mathbb{R}^{3}$ plus a plane, $H_{\infty}$, of points at infinity. Choose a projective transformation $\Psi: R P^{3} \rightarrow R P^{3}$ such that $\Psi\left(x_{0}\right) \in H_{\infty}$. Let $H=\Psi^{-1}\left(H_{\infty}\right)$. By breaking up the characteristic path in (3.2.1) into a finite number of paths, if necessary, we can assume that $H$ contains none of the lines $l(s)$ in the path. Those parts of $W$ and supp $f$ that are far from the union of all $l(s)$ are clearly irrelevant to this argument, so we can assume supp $f$ and $W$ do not meet $H$; thus we can assume $\Psi$ maps $W$ into an analytic surface $\Psi(W)$ in $\mathbb{R}^{3}$ and that $\Psi$ maps $f$ into a distribution $\tilde{f} \in \mathscr{E}^{\prime}\left(\mathbb{R}^{3}\right)$. Since $\Psi$ is real analytic and preserves the order of contact, the hypotheses of Proposition 3.2.1 hold on $\tilde{f}$, $\Psi(W)$, and the Radon transform induced by $\Psi$ on $R_{\mu}$, thus the path of lines $\Psi(l(s))$ has pivot point at infinity.

We now define "cones" of lines in $Y_{0}$ near $l(s)$ that will be used to eat away at the support of $f$ and thus prove (3.2.1). We assume $W$ is tangent to the $x_{1}-x_{3}$ plane at $w_{0}=(0,0,-d)$ and use the equation $y_{2}=h\left(y_{1}, y_{3}\right)$ to define $W$ near $w_{0}$, where $h(0,-d)=0=\left[\partial h / \partial y_{1}\right](0,-d)=\left[\partial h / \partial y_{3}\right](0,-d)$. In this proof, points of tangency to $W$ will be written $\left(y_{1}, h\left(y_{1}, y_{3}\right), y_{3}\right)$ and points on lines will be written $\left(x_{1}, x_{2}, x_{3}\right)$. We can assume that $a=0$ in (3.2.1), that $l(0)=\ell_{0}$ is the $x_{3}$-axis, and that $l(s)$ lies in the plane $x_{1}=s$. The curvature assumption given in the definition of type I complex implies

$$
\frac{\partial^{2} h}{\partial y_{3}^{2}}(0,-d) \neq 0 \text {. }
$$

The set of lines tangent to $W$ and parallel to the $x_{2}-x_{3}$ plane can be locally parametrized as follows. Let $l\left(y_{1}, \alpha\right)$ be the line in $Y$ that lies in the plane $x_{1}=y_{1}$ and is parallel to $(0, \alpha, 1)$. The $y_{3}$-coordinate of the point of tangency to $W$ of this line satisfies

$$
\frac{\partial h}{\partial y_{3}}\left(y_{1}, y_{3}\right)=\alpha .
$$

By (3.2.2) and the Implicit Function Theorem, there is a constant $C>0$ such that for any $\left|y_{1}\right| \leq 2 C$ and $|\alpha| \leq C,(3.2 .3)$ has an analytic solution $y_{3}=$ $y_{3}\left(y_{1}, \alpha\right)$.

Let $\varepsilon(s)$ be a smooth positive function with negative derivative for all $s$. For $s$ near zero define $D(s)$ to be the "cone" of all lines $l\left(y_{1}, \alpha\right)$ satisfying

$$
\left(y_{1}-s\right)^{2}+\alpha^{2} \leq \varepsilon^{2}(s) \text {. }
$$

Assume that $\varepsilon(s)$ is so small that for each $s \in[0, C], \varepsilon(s) \leq C$. This will ensure that the parameterization of lines $l\left(y_{1}, \alpha\right)$ will be valid for all $\left(y_{1}, \alpha\right)$ satisfying (3.2.4). By making $\varepsilon(s)$ sufficiently small we can assume that the nonsmooth part of $\partial D(s)$ (near $W$ ) does not meet $\operatorname{supp} f$ and that for all points $\left(x_{1}, x_{2}, x_{3}\right)$ in $(\operatorname{supp} f) \cap D(s)$,

$$
x_{3} \neq y_{3}\left(x_{1}, \alpha\right) \text { if }\left(x_{1}-s\right)^{2}+\alpha^{2} \leq \varepsilon^{2}(s) .
$$

Finally, we can assume that $D(0)$ does not intersect $\operatorname{supp} f$. Let

$$
\bar{s}=\sup \left\{s_{1} \in[0, C] \mid D(s) \cap \operatorname{supp} f=\varnothing \text { for } 0 \leq s<s_{1}\right\} .
$$


The set in (3.2.6) is nonempty as $D(0)$ does not meet supp $f$. Assume $\bar{s}<C$. Then $D(\bar{s})$ meets supp $f$ at some point $\bar{x}$. By the definition of $\bar{s}, \bar{x}$ must be a boundary point of $D(\bar{s})$. Let $\bar{w}$ be the point of intersection of $W$ and the line $\bar{\ell}$ in $D(\bar{s})$ containing $\bar{x}$.

Lemma 3.2.2. No conormal $\bar{\xi}$ to $\partial D(\bar{s})$ at $\bar{x}$ is conormal to $\Sigma_{\bar{x}}$ along $\bar{\ell}$.

Assuming Lemma 3.2.2 for the moment we complete the proof of Proposition 3.2.1. As $R_{\mu} f=0$ for all lines in a neighborhood of $\bar{\ell}$ and $\bar{\xi}$ is not conormal to $\Sigma_{\bar{x}}$ along $\bar{\ell}$, Proposition 3.1.1 shows that $(\bar{x}, \bar{\xi}) \notin W F_{A}(f)$. As $\bar{\xi}$ is conormal to $\partial D(\bar{s})$ at $\bar{x}$ and $f=0$ to one side of this boundary, Hörmander's Theorem 8.5.6 [14] implies that $f=0$ in a neighborhood of $\bar{x}$. This proves $\bar{s}=C$. This argument can clearly be repeated so that after a finite number of steps one concludes $\bar{s}=b$.

Proof of Lemma 3.2.2. The basic idea is that $\mathscr{W}=\bigcup_{0 \leq s \leq \bar{s}} D(s)$ is a wedge with decreasing width because $\varepsilon(s)$ strictly decreases. The conormal $\bar{\xi}$ to $D(\bar{s})$ at $\bar{x}$ is a conormal to this wedge. If $\bar{\xi}$ were also conormal to $W$ at $\bar{w}$, then the width of the wedge $\mathscr{W}$ would not be decreasing at $\bar{s}$. The rigorous proof is as follows.

The proof is done in two steps. First we find conditions on $\bar{x}=\left(\bar{x}_{1}, \bar{x}_{2}, \bar{x}_{3}\right)$ for conormals to $\partial D(s)$ at $\bar{x}$ to be conormal to $\Sigma_{\bar{x}}$ along $\bar{\ell}$ (equivalently: to be conormal to $W$ at $\bar{w})$. The line $l\left(y_{1}, \alpha\right)$ has equation

$$
\begin{gathered}
x_{1}=y_{1}, \quad x_{2}=H\left(\alpha, y_{1}, x_{3}, y_{3}\left(y_{1}, \alpha\right)\right), \quad \text { where } \\
H\left(\alpha, y_{1}, x_{3}, y_{3}\right):=\alpha\left(x_{3}-y_{3}\right)+h\left(y_{1}, y_{3}\right) .
\end{gathered}
$$

Near $\bar{x}$, the surface $\partial D(\bar{s})$ is generated by the one-parameter family of lines $l\left(y_{1}(t), \alpha(t)\right)$, where

$$
y_{1}(t):=\bar{s}+\varepsilon(\bar{s}) \cos t, \quad \alpha(t):=\varepsilon(\bar{s}) \sin t \text { for } t \in[0,2 \pi] .{ }^{1}
$$

Hence the intersection of $\partial D(\bar{s})$ with the plane $x_{3}=\bar{x}_{3}$ can be parameterized by $x_{1}=y_{1}(t) ; x_{2}=x_{2}(t):=H\left(\alpha(t), y_{1}(t), \bar{x}_{3}, y_{3}\left(y_{1}(t), \alpha(t)\right)\right) ; x_{3}=\bar{x}_{3}$. Since $\partial H / \partial y_{3}=0$ for $y_{3}=y_{3}\left(y_{1}, \alpha\right)$ by (3.2.3), we see

$$
x_{2}^{\prime}(t)=\alpha^{\prime}(t)\left(\bar{x}_{3}-y_{3}\right)+\partial h / \partial y_{1} y_{1}^{\prime}(t) .
$$

Let $\bar{x}$ be on the line parameterized by $\bar{t}$ in (3.2.8). If the normal to $\partial D(\bar{s})$ at $\bar{x}$ were normal to $W$ at $\bar{w}$, then $\left(y_{1}^{\prime}(\bar{t}), x_{2}^{\prime}(\bar{t}), 0\right) \cdot\left(-\partial h / \partial y_{1}, 1,-\partial h / \partial y_{3}\right)=$ 0 . This would imply that $x_{2}^{\prime}(\bar{t})-y_{1}^{\prime}(\bar{t}) \partial h / \partial y_{1}=0$. But because of (3.2.9), $0=\alpha^{\prime}(\bar{t})\left[\bar{x}_{3}-y_{3}\left(y_{1}(\bar{t}), \alpha(\bar{t})\right)\right]$. Because of $(3.2 .5), \alpha^{\prime}(\bar{t})=0$ so $\bar{t}=\pi / 2,3 \pi / 2$. This corresponds to lines $l(\bar{s}, \pm \varepsilon(\bar{s}))$ and points

$$
\bar{x} \text { with } \bar{x}_{1}=\bar{s}
$$

on the "left" and "right" ends of $\partial D(\bar{s})$.

Second we show points (3.2.10) are not in the boundary of the wedge $\mathscr{W}$. Let $g\left(x_{1}, \alpha, s\right)=\left(x_{1}-s\right)^{2}+\alpha^{2}-\varepsilon^{2}(s)$. Then $\frac{\partial g}{\partial s}(\bar{s}, \pm \varepsilon(\bar{s}), \bar{s})=-2 \varepsilon(\bar{s}) \varepsilon^{\prime}(\bar{s})>0$. As $g(\bar{s}, \pm \varepsilon(\bar{s}), \bar{s})=0$, for some $s_{0}<\bar{s}, g\left(\bar{s}, \pm \varepsilon(\bar{s}), s_{0}\right)<0$. This shows that if $\bar{x}$ satisfies (3.2.10), then it is inside $D\left(s_{0}\right)$. Since $s_{0}<\bar{s}$, this contradicts the definition of $\bar{s}$.

${ }^{1}$ By (3.2.3) and (3.2.5) $\partial x_{2} / \partial \alpha$ given from (3.2.7) has the proper sign so that points near $\bar{x}$ on $l\left(y_{1}(t), \alpha(t)\right)$ will be in $\partial D(\bar{s})$. 
Proposition 3.2.3. Assume $W, Y_{0}$, and $f$ satisfy the assumptions of Theorem 2.1. Then any two lines in $Y_{0}$ can be joined in $Y_{0}$ by a finite number of characteristic paths with pivot points away from supp $f$.

Proof. For $\ell \in Y_{0}$ define $E(\ell)$ to be the set of all lines in $Y_{0}$ that can be joined with $\ell$ by a finite sequence of characteristic paths with pivot points away from $\operatorname{supp} f$ and $W$. We will show $E(\ell)$ contains a neighborhood of $\ell$. This implies the lemma; for if $\Gamma$ is a path in $Y_{0}$ joining two arbitrary lines $\ell_{0}$ and $\ell_{1}$ in $Y_{0}$, the collection $\{E(\ell) \mid \ell \in \Gamma\}$ forms an open cover of $\Gamma$, so an application of the Heine-Borel Theorem gives a finite subcovering, completing the proof.

Let $\mathscr{P}$ be a plane perpendicular to $\ell_{0}$ and away from $\operatorname{supp} f$, and let $p_{0}$ be the point of intersection of $\ell_{0}$ and $\mathscr{P}$. Let $\sigma$ be the map that associates to any line $\ell$ in $Y$ near $\ell_{0}$ its point of intersection with $\mathscr{P}$. Then $\sigma$ is a smooth fibering of a neighborhood of $\ell_{0}$ and the fiber above $x \in \mathscr{P}$ is the characteristic path with pivot point $x$. We will prove:

$$
\begin{aligned}
& \text { projections under } \sigma \text { of characteristic paths connect } p_{0} \text { with } \\
& \text { every point in some neighborhood, } N \text {, of } p_{0} \text { in } \mathscr{P} \text {. }
\end{aligned}
$$

This statement will finish the proof of the proposition by allowing one to connect $\ell_{0}$ to any nearby line by at most two characteristic paths.

To prove (3.2.11), we first construct a smooth foliation of $\mathscr{P}$ near $p_{0}$ by projections of characteristic paths under $\sigma$. Let $\mathscr{Q}$ be another plane perpendicular to $\ell_{0}$ and not intersecting supp $f$. Let $q_{0}$ be the point of intersection of $\ell_{0}$ with $\mathscr{Q}$. Choose a small segment $S$ through $q_{0}$ on $\mathscr{Q}$ that is perpendicular to the tangent plane of $W$ at $w_{0}$, the point of tangency of $\ell_{0}$ with $W$. Each $q \in S$ is the pivot point of a characteristic path. If $\lambda_{q}$ is the projection under $\sigma$ of the characteristic path with pivot point $q$, then the $\lambda_{q}$ for $q \in S$ give a one-parameter family of smooth curves in $\mathscr{P}$ that is a foliation of a neighborhood of $p_{0}$. (This can be shown by proving that $(q, t) \rightarrow \lambda_{q}(t)$ for $q \in S$ and $t$ a real parameter define local coordinates on $\mathscr{P}$.) Let $v_{0}$ be a tangent vector at $p_{0}$ to the curve $\lambda_{q_{0}}$. The vector $v_{0} \neq 0$ as $q_{0} \neq p_{0}$.

We now construct another characteristic path with projection that is transversal to the family $\lambda_{q}$ at $p_{0}$. Let $\alpha$ be the curve traced out on $\mathscr{Q}$ by the characteristic path of lines with pivot point $p_{0}$. The tangent line at $p_{0}$ to the curve in $\mathscr{P}$ traced out by pivoting at $q \in \alpha$ is the intersection of $\mathscr{P}$ and the tangent plane to $W$ through the line $\overline{p_{0} q}$ connecting $p_{0}$ and $q$. The only way that all such tangent lines at $p_{0}$ for different $q \in \alpha$ can be parallel to $v_{0}$ is if all the corresponding tangent planes to $W$ of lines $\overline{p_{0} q}$ are parallel and hence the same plane. This would contradict the assumption that every line in $Y_{0}$ is tangent to $W$ along a tangent plane that is tangent to $W$ in only a discrete set of points. Therefore, for some $q \in \alpha$, the curve on $\mathscr{P}$ traced out by pivoting at $q$ is transversal to $v_{0}$. This transversal curve allows one to connect $p_{0}$ with any curve in the foliation that is sufficiently near $p_{0}$. The curve itself is the projection of a characteristic path (the one pivoting at $q$ ), so since the foliation fills out a neighborhood of $p_{0},(3.2 .11)$ is proved.

Proposition 3.2.1 implies that if $f$ is zero near a line $\ell_{0} \in Y_{0}$ then $f$ is zero near points on lines in every characteristic path starting at $\ell_{0}$. Proposition 3.2.3 implies that $\ell_{0}$ can be connected to any line in $Y_{0}$ by a sequence of characteristic paths. This finishes the proof of Theorem 2.1. 
3.3. Characteristic paths for type II complexes and the proof of Theorem 2.2. The proof follows the general outline of the proof of Theorem 2.1. Let $\ell_{0} \in Y_{0}$ be a line disjoint from supp $f$ and let $\ell_{1} \in Y_{0}$. We will construct a sequence of characteristic paths from $\ell_{0}$ to $\ell_{1}$ in $Y_{0}$ and show supp $f$ meets no line on the path.

A characteristic path with pivot point $x_{0} \in X$ is a smooth path of lines in $\pi_{Y}\left(\pi_{X}^{-1}\left(x_{0}\right)\right)$-a smooth path of lines through $x_{0}$ and meeting $\gamma$. A characteristic path with pivot point at infinity is a path in $Y$ :

$$
l(s)=\ell\left(\theta_{0}, \gamma(s)\right) \text { for fixed } \theta_{0}, a \leq s \leq b .
$$

Proposition 3.3.1. Assume that the hypotheses of Theorem 2.2 hold for $X, \gamma$, and $Y_{0}$. Let $f \in \mathscr{E}^{\prime}(X)$ and assume $R_{\mu} f=0$ on $Y_{0}$. Let $l:[a, b] \rightarrow Y_{0}$ be $a$ characteristic path and assume $l(a)$ does not meet supp $f$ and the pivot point of the path $l(s)$ is disjoint from supp $f$. Then

$$
l(s) \cap \operatorname{supp} f=\varnothing \quad \text { for } a \leq s \leq b .
$$

Proof. As in the proof of Proposition 3.2.1, we can assume the pivot point is at infinity. For such paths we use the family of circular cones

$$
D(s)=\bigcup\left\{\ell(\theta, \gamma(s))\left|\theta \in \mathbf{S}^{2},\right| \theta-\theta_{0} \mid \leq \varepsilon(s)\right\} \text { for } s \in[a, b],
$$

where $\varepsilon(s)$ is a positive function with negative derivative such that $D(a)$ is so small that it does not intersect supp $f$, and for all $s \in[a, b], D(s)$ contains only lines in $Y_{0}$. Furthermore $\varepsilon$ must decrease fast enough so that in each planar cross-section $C$ of supp $f$ that is perpendicular to $\theta_{0}, D(s) \cap C$ has decreasing radius as $s$ increases. This can be done because supp $f$ is compact and lies away from $\gamma(\mathbb{R})$.

One way is as follows: For some $\rho>0$ the tube about $\gamma$ of radius $\rho$ is disjoint from $\operatorname{supp} f$. Let $\mathscr{P}_{0}$ be the plane perpendicular to $\theta_{0}$ and through $\gamma(a)$. Assume that for $s>a$ and $s$ sufficiently near $a, \gamma(s)$ and $\gamma^{\prime}(s)$ lie "above" $\mathscr{P}_{0}$ (that is, $(\gamma(s)-\gamma(a)) \cdot \theta_{0}$ and $\gamma^{\prime}(s) \cdot \theta_{0}$ both have the same sign or are 0$)$. Let $\mathscr{P}_{1}$ be the plane $\rho / 2$ units "below" $\mathscr{P}_{0}$. Let $g(s)$ be the projection of $\gamma(s)$ onto $\mathscr{P}_{1}$. Let $e(s)$ be a positive decreasing function for $s \geq a$. Define $\varepsilon(s)$ and $D(s)$ so that $D(s) \cap \mathscr{P}_{1}$ is the disc about $g(s)$ of radius $e(s) \quad(e(s)$ must be so small that the other requirements on $D(s)$ are satisfied, too). Because $D(s) \cap \mathscr{P}_{1}$ does not touch supp $f$ locally near $\gamma(a)$, and since $\gamma$ and $\gamma^{\prime}$ lie above $\mathscr{P}_{0}, \varepsilon$ decreases fast enough so that the radius of $D(s) \cap C$ decreases for any cross-section $C$ of supp $f$ perpendicular to $\theta_{0}$. This allows one to move $D(a)$ to $D\left(a_{1}\right)$ for some $a_{1}>a$ and have the radius of $D(s) \cap C$ decrease as $s$ increases to $a_{1}$. This process can be continued to $b$ because $\rho$ is fixed, $\gamma^{\prime}$ is never parallel to $\theta_{0}$, and $\gamma([a, b])$ is compact.

Because the radii of the cross-sections are decreasing, as in the proof of Proposition 3.2.1, no cotangent vector conormal to $\gamma^{\prime}(s)$ is conormal to the boundary of $\bigcup_{a \leq s \leq s} D(s)$. Now Proposition 3.1.1 and Hörmander's Theorem 8.5.6 [14] can be used to finish the proof.

Proposition 3.3.2. Assume that the hypotheses of Theorem 2.2 hold on $\gamma$ and $Y_{0}$. Then any two lines in $Y_{0}$ can be joined by a finite number of characteristic paths. 
Proof. We use the notation of Proposition 3.2.3. The set of curves $\gamma_{q}$ is a foliation on $\mathscr{P}$ near $p_{0}$ because the curves $\gamma_{q}$ are defined in terms of local coordinates on $Y$. The fact that $Y_{0}$ contains no line in any plane containing $\gamma$ (if $\gamma$ is a plane curve) is used to construct a curve in the plane $\mathscr{P}$ transversal to the foliation $\gamma_{q}$. The rest of the proof is essentially the same as the proof of Proposition 3.2.3.

The conclusion of Theorem 2.2 is true if lines tangent to $\gamma$ are included in $Y_{0}$. Under the hypotheses of Theorem 2.2, $\gamma$ is not a line; so, if $\ell$ is a line tangent to $\gamma$ and $x \in \ell \backslash \gamma$, then $x$ is also on lines in $Y$ that are arbitrarily close to $\ell$ and that are not tangent to $\gamma$. Thus, Theorem 2.2 can be applied to this connected set of nontangent lines to eat away at supp $f \cap \ell$.

3.4. Proof of Theorem 2.3. We prove this by reducing to Theorem 2.2 using an affine motion of $R P^{3}$. The curve of directions on $\mathbf{S}^{2}, \theta(t)$ for $t \in \mathbf{S}^{1}$, can be viewed as a curve in the plane at infinity in $R P^{3}$. Each line with direction vector $\theta(t)$ intersects the same point on this curve at infinity. There is an affine motion, $\Psi$, of $R P^{3}$ that moves this curve at infinity (with at most a finite number of points removed) to an analytic plane curve, $\gamma$, in $\mathbb{R}^{3}$. As $\theta\left(\mathbf{S}^{1}\right)$ is not a great circle of $\mathbf{S}^{2}, \gamma$ is not a line and so $\operatorname{supp}\left(f \circ \Psi^{-1}\right)$ does not meet any plane containing $\gamma$. As $f$ has compact support, $\Psi$ can be chosen so $\operatorname{supp}\left(f \circ \Psi^{-1}\right)$ stays in $\mathbb{R}^{3}$. Now Theorem 2.2 applied to $\gamma$ and $f \circ \Psi^{-1}$ can be used to prove Theorem 2.3.

\section{EXAMPLeS}

Counterexamples are given to Theorems 2.1, 2.2, and 2.3 if their geometric assumptions on the line complexes are not satisfied. An injective Radon transform on a nonadmissible complex is given.

The Radon transform in Examples 4.1-4.2 is the Radon transform with measure $\mu=1, R=R_{1}$. The function used in the counterexamples will be (4.1)

$$
f\left(x_{1}, x_{2}, x_{3}\right)= \begin{cases}1 & \text { if }\left(x_{1}, x_{2}, x_{3}\right) \in[-500,500] \times[-1,0] \times[2,3], \\ -1 & \text { if }\left(x_{1}, x_{2}, x_{3}\right) \in[-500,500] \times[-1,0] \times[1,2], \\ 0 & \text { otherwise }\end{cases}
$$

The function's crucial property is that $\left(\left(x_{1}, 0, x_{3}\right),(0,1,0)\right) \in W F_{A}(f)$ for all $\left(x_{1}, x_{3}\right) \in[-500,500] \times[1,3]$. The counterexamples are constructed so that these singularities are not detected by data $\left.R_{\mu} f\right|_{Y_{0}}$.

Example 4.1. This example gives a type I complex $Y$ defined by a surface that is tangent to a plane along a nondiscrete set and for which the conclusion of Theorem 2.1 does not hold.

Let $W$ be a torus that is tangent to the $x_{1}-x_{3}$ plane along a circle, lies in the bottom half space, $x_{3}<0$, and is symmetric about the $x_{2}-x_{3}$ plane. Let $Y$ be the type I complex defined by $W$, let $x^{0}=\left(0,0, x_{3}^{0}\right)$ be one of the two points where the $x_{3}$-axis is tangent to $W$, and let $Y_{0} \subset Y$ be an open connected set of lines sufficiently close to the $x_{3}$-axis and tangent to $W$ near $x^{0}$. The $x_{1}-x_{3}$ plane is tangent to $W$ in a nondiscrete set of points (a circle), but all other hypotheses of Theorem 2.1 hold for $Y_{0}$. However, the conclusion of Theorem 2.1 does not hold because every line in $Y_{0}$ intersects 
$[-500,500] \times[-1,0] \times[2,3]$ in a segment of the same length as its intersection with $[-500,500] \times[-1,0] \times[1,2]$.

Example 4.2. This example gives a type II complex $Y$ defined by a curve (circle) that lies in a plane and for which the conclusion of Theorem 2.2 does not hold. A similar example contradicts the conclusion of Theorem 2.3 if the curve $\theta$ in $S^{2}$ is a great circle.

Let $\gamma$ be a circle in the bottom half of the $x_{1}-x_{3}$ plane and symmetric about the $x_{3}$-axis. Let $Y$ be the type II complex defined by $\gamma$ and let $Y_{0}$ be an open connected subset of $Y$ that is sufficiently close to the $x_{3}$-axis. The support of the function $f$ intersects the plane of $\gamma$ but all other hypotheses of Theorem 2.2 hold. However, the conclusion of Theorem 2.2 does not hold for reasons similar to those given in Example 4.1. Similarly, one can construct a counterexample to the conclusion of Theorem 2.3 using the function $f$ above and the type III complex $Y$ defined by the great circle of $\mathbf{S}^{2}$ parallel to the $x_{1}-x_{3}$ plane.

Example 4.3. This example provides a Radon transform on a nonadmissible line complex $Y$ for which Helgason's support theorem holds for functions of compact support. This is a degree one complex as defined in $\S 6$ of [9] but it is not admissible because Gelfand's admissibility condition, (3.1.3), fails.

The complex, $Y$, we will consider will be parameterized by $y=\left(y_{1}, y_{2}, \theta_{1}\right)$; the line $l(y)$ will be defined to be the line intersecting $\left(y_{1}, y_{2}, 0\right)$ and parallel to $\left(\theta_{1}, \theta_{2}(y), 1\right)$, where

$$
\theta_{2}(y)=y_{1}
$$

This example (with canonical measures) was described in the complex category by Gelfand and Graev [6] (see also [9, pp. 231-237] for lines in $\mathbb{R}^{3}$ ).

Theorem 4.1. Let $Y$ be the line complex defined in (4.2), and let $\mu(x, \ell)$ be a real analytic function on $Z$ (see (2.1)) that is never zero. Let $f \in \mathscr{E}^{\prime}\left(\mathbb{R}^{3}\right)$. Assume $R_{\mu} f$ is zero for lines in $Y$ that lie outside of the convex compact set $K$. Then $f$ is supported in $K$.

Proof of Theorem 4.1. We will only outline the microlocal analysis for this Radon transform as the calculations are similar to those in the admissible case. The set $Z$ of $(2.1)$ is defined by

$$
x_{1}=y_{1}+x_{3} \theta_{1} \quad \text { and } \quad x_{2}=y_{2}+x_{3} y_{1},
$$

and $\Lambda$ is the set of all $(x, \xi, y, \eta)$ such that

$$
\begin{array}{ll}
\xi_{1}=-a_{1}, & \eta_{1}=a_{1}+x_{3} a_{2}, \\
\xi_{2}=-a_{2}, & \eta_{2}=a_{2}, \\
\xi_{3}=a_{1} \theta_{1}+a_{2} y_{1}, & \eta_{3}=a_{1} x_{3},
\end{array}
$$

where $a_{1}$ and $a_{2}$ are not both zero. It follows that $\Lambda$ is a subset of $T^{*}(X) \backslash 0 \times T^{*}(Y) \backslash 0$, and $R_{\mu}$ is an analytic Fourier integral operator associated with $\Lambda$. Let $\Lambda_{0}$ be the set of all $(x, \xi, y, \eta) \in \Lambda$ satisfying

$$
\xi_{2} \neq 0 \text { and } \xi_{1}-\xi_{2} x_{3} \neq 0 \text {. }
$$

It follows that $\pi_{X}: \Lambda_{0} \rightarrow T^{*} X$ is an injective immersion, and all $(x, \xi) \in$ $T^{*}(X) \backslash 0$ that satisfy $(4.5)$ are in $\pi_{X}\left(\Lambda_{0}\right)$. This can be seen by solving for 
$(y, \eta)$ in (4.3) and (4.4) using (4.5). To prove $\pi_{Y}: \Lambda_{0} \rightarrow T^{*} Y$ is two-to-one, one uses (4.4) to show $x_{3}$ satisfies

$$
\eta_{2} x_{3}^{2}-\eta_{1} x_{3}+\eta_{3}=0
$$

Because of the first condition in (4.5), $\eta_{2} \neq 0$. Thus (4.6) has two distinct solutions for $x_{3}$ if and only if $\eta_{1}^{2}-4 \eta_{2} \eta_{3} \neq 0$; by the relation between $\eta$ and $\xi$ given in (4.4) the latter expression is equal to $\left(\xi_{1}-\xi_{2} x_{3}\right)^{2}$ on $\Lambda$. Now the second condition in (4.5) implies there are two distinct solutions to (4.6) on $\Lambda_{0}$ and so $\pi_{Y}$ is two-to-one on $\Lambda_{0}$. It is important to note that if $(y, \eta) \in \pi_{Y}\left(\Lambda_{0}\right)$ then the two points $(x, \xi)$ and $(\hat{x}, \hat{\xi})$ associated to $(y, \eta)$ in $\Lambda_{0}$ satisfy $\hat{x}_{3}=\xi_{1} / \xi_{2}$ and the dual relationship $x_{3}=\hat{\xi}_{1} / \hat{\xi}_{2}$. The points $(\hat{x}, \hat{\xi})$ and $(x, \xi)$ will be called dual points. The calculation showing $\pi_{Y}$ is a local diffeomorphism on $\Lambda_{0}$ is straightforward. These comments and the calculus of analytic Fourier integral operators prove the following lemma.

Lemma 4.2. Let $(x, \xi) \in T^{*}\left(\mathbb{R}^{3}\right)$ satisfy (4.5). Let $\ell_{0}$ be the line in $Y$ with $(x, \xi) \in N^{*}\left(\ell_{0}\right)$. Let $f \in \mathscr{E}^{\prime}\left(\mathbb{R}^{3}\right)$ and assume $R_{\mu} f=0$ for all lines in $Y$ sufficiently near $\ell_{0}$. Assume also that $\hat{x}$ is not in supp $f$, where $(\hat{x}, \hat{\xi})$ is the dual point associated to $(x, \xi)$. Then $(x, \xi) \notin W F_{A}(f)$.

Note that the assumption $\hat{x} \notin \operatorname{supp} f$, in Lemma 4.2 prevents singularities at $\hat{x}$ and $x$ from canceling when evaluating $R_{\mu} f$; precisely, in the proof we replace $R_{\mu}$ by a restricted operator, $S_{\mu}$, for which the analogous projection to $\pi_{Y}$ is injective and for which $S_{\mu} f=0$.

To finish the proof of Theorem 4.1 we eat away at supp $f \backslash K$ by enclosing supp $f \cup K$ in slightly deformed balls. Let $a=\left(a_{1}, a_{2}, a_{3}\right) \in \mathbb{R}^{3}$ be fixed and let $r>0$; now define $S_{r}$ to be the sphere centered at $a$ of radius $r$. For small positive $\varepsilon$ let $C$ be the set of $x \in S_{r+\varepsilon}$ such that for some covector $\xi \in N_{x}^{*}\left(S_{r+\varepsilon}\right), \quad(x, \xi)$ does not satisfy the second condition in (4.5). Then $C$ consists of a curve that "spirals" between the south and north poles of $S_{r+\varepsilon}$. Let $\mathscr{P}_{t}$ be the horizontal plane $x_{3}=t$ and let $D_{r}$ be the union of the convex hulls of $\left(C \cup S_{r}\right) \cap \mathscr{P}_{t}$ for $t \in\left[a_{3}-r+\varepsilon, a_{3}+r-\varepsilon\right]$. The set $D_{r}$ consists of a deformed ball between the flat top, $T$, and the flat bottom, $B$. Finally, let $L$ be the intersection of $\partial D_{r}$ with the plane through $a$ and parallel to the $x_{1}-x_{3}$ plane; $\varepsilon$ can be chosen so small that $L$ is a longitudinal great circle of $S_{r}$ flattened at the top and bottom. Therefore, covectors $(x, \xi)$ conormal to $\partial D_{r}$ along points $x \in L$ have $\xi_{2}=0$.

We claim that all points $(x, \xi) \in N^{*}\left(\partial D_{r} \backslash(C \cup T \cup B \cup L)\right)$ satisfy (4.5). This is true because either such points are on $S_{r}$ and away from "bad" points not satisfying (4.5) or they are near enough to $C$ so that they lie on horizontal lines between $S_{r}$ and $C$ and have conormals $\xi$ satisfying $\xi_{1} / \xi_{2}<x_{3}$ or $\xi_{1} / \xi_{2}>x_{3}$ (this second statement is true as $C$ "juts" out from $S_{r}$ along tangent lines to $\left.S_{r}\right)$.

Now assume the center $a$ and radius $r$ of $D_{r}$ are chosen so that supp $f \cup K$ $\subset D_{r}$ and so that supp $f \cup K$ is in one of the open half spaces determined by the plane $x_{2}=a_{2}$. Therefore $L$ does not meet supp $f$. The radius $r$ can be chosen so large that supp $f$ does not meet $T$ or $B$ as well. Therefore, if supp $f \backslash K$ meets $\partial D_{r}$ then it must meet at a point $x_{0} \in C$ (if $x_{0} \notin C$, then Lemma 4.2 and Hörmander's Theorem 8.5.6 [14] show $x_{0} \notin \operatorname{supp} f$ ). As $D_{r}$ 
is convex and is "pointed" at $x_{0}$, there is a plane through $x_{0}$ tangent to $D_{r}$ in conormal direction $\xi_{0}$ where $\left(x_{0}, \xi_{0}\right)$ does satisfy $(4.5)$. Thus Hörmander's theorem and Lemma 4.2 provide a contradiction. Since such sets $D_{r}$ can get arbitrarily close to $K, \operatorname{supp} f \subset K$.

\section{BIBLIOGRAPHY}

1. J. E. Björk, Rings of differential operators, North-Holland, Amsterdam, 1979.

2. J. Boman, Uniqueness theorems for generalized Radon transforms, Constructive Theory of Functions '84, Sofia, 1984, pp. 173-176.

3. J. Boman and E. T. Quinto, Support theorems for real analytic Radon transforms, Duke Math. J. 55 (1987), 943-948.

4. D. V. Finch, Cone beam reconstructions with sources on a curve, SIAM J. Appl. Math. 45 (1985), 665-673.

5. D. V. Finch, Uniqueness of the attenuated X-ray transform in the physical range, Inverse Problems 2 (1986), 197-203.

6. I. M. Gelfand and M. I. Graev, Integral transformations connected with straight line complexes in complex affine space, Dokl. Akad. Nauk SSSR 2 (1961), 809-812.

7. I. M. Gelfand, M. I. Graev, and N. Vilenkin, Generalized functions, Vol. 5, Academic Press, New York, 1966.

8. J. Globevnik, A support theorem for the X-ray transform, J. Math. Anal. Appl. 165 (1992), 284-287.

9. A. Greenleaf and G. Uhlmann, Non-local inversion formulas in integral geometry, Duke Math. J. 58 (1989) 205-240.

10. V. Guillemin, On some results of Gelfand in integral geometry, Proc. Sympos. Pure Math., vol. 43, Amer. Math. Soc., Providence, R.I., 1985, 149-155.

11. V. Guillemin and S. Sternberg, Geometric asymptotics, Amer. Math. Soc., Providence, R.I., 1977.

12. S. Helgason, Groups and geometric analysis, Academic Press, New York, 1984.

13. L. Hörmander, Fourier integral operators. I, Acta Math. 127 (1971), 79-183.

14. _ . The analysis of linear partial differential operators. I, Springer, New York, 1983.

15. A. Kaneko, Introduction to hyperfunctions, Kluwer, New York, 1989.

16. A. Markoe and E. T. Quinto, An elementary proof of local invertibility for generalized and attenuated Radon transforms, SIAM J. Math. Anal. 16 (1985), 1114-1119.

17. E. T. Quinto, The dependence of the generalized Radon transform on defining measures, Trans. Amer. Math. Soc. 257 (1980), 331-346.

18. _., Real analytic Radon transforms on rank-one symmetric spaces, Proc. Amer. Math. Soc. (to appear).

19. M. Sato, T. Kawai, and M. Kashiwara, Hyperfunctions and pseudodifferential equations, Lecture Notes in Math., vol. 287, Springer, New York, 1973, pp. 265-529.

20. D. C. Solmon, The X-ray transform, J. Math. Anal. Appl. 56 (1976), 61-83.

21. F. Treves, Introduction to pseudodifferential and Fourier integral operators. I, Plenum Press, New York, 1980.

Department of Mathematics, Stockholm University, S-11385 Stockholm, Sweeden

E-mail address: jabo@matematik.su.se

Department of Mathematics, Tufts University, Medford, Massachusetts 02155, USA.

E-mail address: equinto@math.tufts.edu 